\title{
Quasi-isomorphisms of cluster algebras and the combinatorics of webs (extended abstract)
}

\author{
Chris Fraser $\|^{\dagger}$ \\ Department of Mathematics, University of Michigan, Ann Arbor 48109 USA
}

\begin{abstract}
We provide bijections between the cluster variables (and clusters) in two families of cluster algebras which have received considerable attention. These cluster algebras are the ones associated with certain Grassmannians of $k$-planes, and those associated with certain spaces of decorated $\mathrm{SL}_{k}$-local systems in the disk in the work of Fock and Goncharov. When $k$ is 3 , this bijection can be described explicitly using the combinatorics of Kuperberg's basis of non-elliptic webs. Using our bijection and symmetries of these cluster algebras, we provide evidence for conjectures of Fomin and Pylyavskyy concerning cluster variables in Grassmannians of 3-planes. We also prove their conjecture that there are infinitely many indecomposable nonarborizable webs in the Grassmannian of 3-planes in 9-dimensional space.

Résumé. Nous fournissons des bijections entre les variables amassées de deux familles d'algébres amassées qui ont reçu de l'attention considérable. Ces algébres amassées sont celles associées á certaines grassmanniennes de $k$-plans et celles associées á certains espaces de $\mathrm{SL}_{k}$-systèmes locaux décorés sur le disque au sens de Fock et Goncharov. Lorsque $k=3$, cette bijection peut être décrite explicitement en utilisant la combinatoire de la base de Kuperberg des toiles réduites. Notre bijection, ainsi que certaines symétries de ces algébres amassées, fournit de l'évidence pour les conjectures de Fomin et Pylyavskyy concernant les variables amassées dans les grassmanniennes de dimension 3. Nous démontrons aussi leur conjecture qu'il existe une infinité de toiles nonarborizables indécomposables dans la grassmannienne de 3 -plans dans un espace de dimension 9.
\end{abstract}

Keywords. cluster algebra, web, quasi-isomorphism, Grassmannian

\section{Introduction}

Cluster algebras are a rich class of commutative algebras, introduced by Fomin and Zelevinsky [7] in order to axiomatize constructions (such as canonical bases and totally positive parts) arising in the representation theory of Lie groups. Speaking briefly, a cluster algebra is a commutative ring endowed with a special set of generators (called cluster variables) grouped into overlapping sets of the same size (called clusters). Beginning with a single initial cluster, there is a recursive procedure for producing the remaining clusters by iterating certain transformations called mutations.

\footnotetext{
${ }^{\dagger}$ Email: cmfra@umich. edu. Supported by a graduate fellowship from the National Physical Science Consortium and in part by NSF grant DMS-1361789.
}

1365-8050 @ 2016 Discrete Mathematics and Theoretical Computer Science (DMTCS), Nancy, France 
One of the most familiar examples of a cluster algebra is the coordinate ring $\mathbb{C}[\hat{\operatorname{Gr}}(2, n)]$ of the affine cone over the Grassman variety $\operatorname{Gr}(2, n)$ of 2 -dimensional subspaces in an $n$-dimensional complex vector space. This is the ring generated by the Plücker coordinates $\Delta_{i j}$ where $1 \leq i<j \leq n$. The combinatorics of the clusters is the Catalan combinatorics of triangulations of an $n$-gon: the set of cluster variables (and frozen variables, to be defined below) coincides with the set of Plücker coordinates. Picturing each cluster variable $\Delta_{i j}$ by the arc in the $n$-gon joining vertex $i$ to vertex $j$, the clusters in $\mathbb{C}[\hat{\operatorname{Gr}}(2, n)]$ are exactly the triangulations of the $n$-gon.

There is a well understood generalization of the cluster algebras $\mathbb{C}[\hat{\operatorname{Gr}}(2, n)]$ in which the $n$-gon is replaced by any surface with boundary and marked points [6, 9]). Beyond this class of examples, there are very few instances of cluster algebras in which the entire combinatorics of clusters is known. Fomin and Pylyavskyy [4] have initiated a study of the cluster algebras $\mathbb{C}[\hat{\mathrm{G}} \mathrm{r}(3, n)]$ using a description in terms of planar diagrams called non-elliptic webs. There is a conjectural description of the cluster variables (as indecomposable non-elliptic web invariants that are also arborizable) and of the clusters. For the Grassmannians $\hat{\operatorname{Gr}}(3,5), \hat{\operatorname{Gr}}(3,6), \hat{\mathrm{Gr}}(3,7), \hat{\mathrm{Gr}}(3,8)$ there are only finitely many cluster variables and the conjectures can be verified by hand. The Grassmannnian $\hat{\operatorname{Gr}}(3,9)$ is an interesting special case because it is of finite mutation type. In particular, there should be a large group of symmetries of the cluster structure on this Grassmannian.

Our main result is a combinatorial realization of such a group of symmetries for $\mathbb{C}[\hat{\mathrm{G}} \mathrm{r}(3,9)]$ using webs. The symmetries arise via the following construction: we produce a quasi-isomorphism of $\mathbb{C}[\mathrm{Gr}(3,9)]$ with a certain Fock-Goncharov cluster algebra $\mathbb{C}\left[\mathrm{Conf}_{6}\left(\mathrm{SL}_{3}\right)\right]$. This quasi-isomorphism induces a bijection between the cluster variables in these two cluster algebras. Certain obvious symmetries for $\mathbb{C}\left[\mathrm{Conf}_{6}\left(\mathrm{SL}_{3}\right)\right]$ produce "hidden" symmetries of $\mathbb{C}[\hat{\mathrm{Gr}}(3,9)]$ under this bijection.

This extended abstract is organized as follows. Section 2 briefly reviews standard cluster algebra definitions and the standard cluster structure on the Grassmannian. Section 3 introduces web combinatorics and the connection to cluster algebras via conjectures of Fomin and Pylyavskyy. We end the section by stating our first two Theorems. Section 4 suggests how these results (and others) are proved, by introducing a quasi-isomorphism between Grassmannian and Fock-Goncharov cluster algebras. This includes a description of the group of symmetries. Section 5 introduces webs for $\mathrm{SL}_{k}$ when $k>3$, and discusses how certain theorems in the previous section extend to this setting, as well as a connection with the twist map on the Grassmannian. We close with a brief discussion of future work.

\section{Cluster algebra preliminaries}

Definition (Ice quiver). An ice quiver is a finite directed graph $Q$ on the the vertex set $[1, n+m]$, without loops or directed 2-cycles. The vertices $n+1, \ldots, n+m$ are called frozen vertices, and we assume that there are no edges between frozen vertices. The vertices $1, \ldots, n$ are called mutable vertices.

Let $k \in[1, n]$ be a mutable index. The operation of quiver mutation in direction $k$ replaces an ice quiver $Q$ by a new quiver $Q^{\prime}=\mu_{k}(Q)$. The quiver $\mu_{k}(Q)$ is obtained from $Q$ by performing three steps:

- For each directed path $i \rightarrow k \rightarrow j$ of length two through $k$ in $Q$, add an arrow $i \rightarrow j$ (do not perform this step if both of $i$ and $j$ are frozen).

- Reverse the direction of all arrows incident to vertex $k$.

- Remove any oriented 2-cycles created in performing the first two steps. 
Definition (Seed). Let $\mathcal{F}$ be a field isomorphic to a field of rational functions in $n+m$ variables. A seed is a pair $(Q, \mathbf{x})$ where $Q$ is an ice quiver on $n+m$ variables, and $\mathbf{x}=\left(x_{1}, \ldots, x_{n} ; x_{n+1}, \ldots, x_{n+m}\right)$ is an extended cluster consisting of elements of $\mathcal{F}$ that are algebraically independent and form a transcendence basis for $\mathcal{F}$. The vertices $x_{n+1}, \ldots, x_{n+m}$ are called frozen variables. The set $\left\{x_{1}, \ldots, x_{n}\right\}$ is called a cluster and its elements are called cluster variables.

Again, we let $k$ be a mutable index. The operation of seed mutation in direction $k$ replaces a seed $(Q, \mathbf{x})$ by a new seed $\left(Q^{\prime}, \mathbf{x}^{\prime}\right)$ where $Q^{\prime}=\mu_{k}(Q)$ and the new cluster variable $x_{k}^{\prime}$ is satisfies an exchange relation

$$
x_{k}^{\prime} x_{k}=\prod_{i \in Q} x_{i}^{\text {number of arrows } i \rightarrow k}+\prod_{i \in Q} x_{i}^{\text {number of arrows } k \rightarrow i} .
$$

The remaining $x_{j}^{\prime}$ are unchanged, i.e. $x_{j}^{\prime}=x_{j}$ if $j \in[1, n+m] /\{k\}$. In particular, the frozen variables are not changed by performing mutations.

Mutating twice in direction $k$ twice in a row is the identity map on seeds.

Definition (Cluster algebra). Starting with a choice of initial seed $\Sigma$, the associated seed pattern $\mathcal{E}$ is the collection of seeds which can be obtained by performing an arbitrary sequence of mutations from $\Sigma$. The cluster algebra associated to such a seed pattern is the $\mathbb{C}$-algebra generated by the cluster variables arising in the seeds of $\mathcal{E}$, along with the frozen varibles.

The collection of seeds in a seed pattern, and therefore the resulting cluster algebra, does not depend on the choice of initial seed. For a given commutative domain $R$, we say that $R$ has a cluster structure if there is a choice of seed $\Sigma$ in the fraction field $\operatorname{Frac}(R)$ for which the resulting cluster algebra is isomorphic to $R$.

Definition (Grassmannian algebras). Let $\left(x_{i j}\right)_{i \in[1, k], j \in[1, n]}$ be a family of $k n$ indeterminates, thought of as the entries of a $k \times n$ matrix. For a subset $S \subset[1, n]$ of size $k$, we let $\Delta_{S} \in \mathbb{C}\left[x_{i j}^{\prime} s\right]$ denote the Plücker coordinate, i.e. the $k \times k$ minor of this matrix whose columns are taken from $S$. We denote by $\mathbb{C}[\hat{\mathrm{Gr}}(k, n)]$ the $\mathbb{C}$-algebra generated by the $\Delta_{S}$ as $S$ ranges over all $k$-subsets of $n$.

The ring in Definition 2 is the coordinate ring of the affine cone $\hat{\operatorname{Gr}}(k, n)$ over the Grassmann manifold $\operatorname{Gr}(k, n)$ of $k$-dimensional subspaces in $\mathbb{C}^{n}$, with respect to the Plücker embedding of $\operatorname{Gr}(k, n)$ in projective space. This ring has a cluster structure which was originally decribed by J. Scott [15]. The frozen variables consist of Plücker coordinates $\Delta_{S}$ whose columns are cyclically consecutive. Every Plücker coordinate is a cluster variable, although there are typically infinitely many additional cluster variables.

\section{Kuperberg's webs}

Definition. A tensor diagram is a finite bipartite graph $T$ with a fixed bipartition into black and white color sets, subject to the following additional conditions. The graph $T$ is drawn in the disk and considered up to isotopy. Certain vertices of $T$ are distinguised as boundary vertices and these vertices reside on the boundary of the disk. Every boundary vertex is black. The remaining vertices reside in the interior of the disk, and all of these interior vertices are trivalent (the boundary vertices can have arbitrary valence, including zero). A tensor diagram is called a $w e b$ if it is planar. 


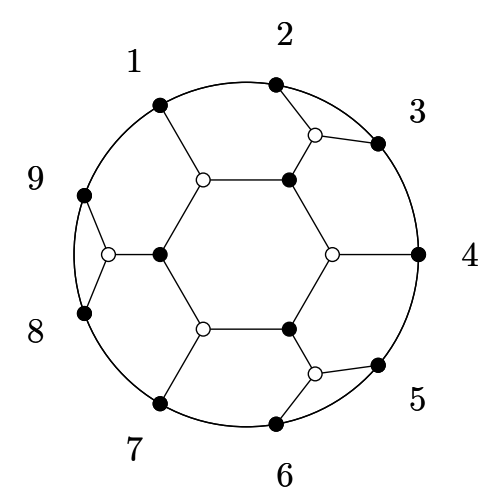

Fig. 1: A web for $\operatorname{Gr}(3,9)$.

Every tensor diagram $T$ with $n$ boundary vertices defines an invariant $[T] \in \mathbb{C}[\hat{\mathrm{Gr}}(3, n)]$ according to the following prescription (cf. [4, Equation (4.1)] ):

$$
[T]=\sum_{\text {proper labelings } \ell}(-1)^{\ell} M(\ell) \in \mathbb{C}\left[x_{i j}\right]_{i \in[1, k], j \in[1, n]}
$$

where

- the sum is over proper labelings $\ell$ of $T$; that is labelings of the edges of $T$ with the numbers $1,2,3$ such that the labels are distinct around each interior vertex,

- the sign $(-1)^{\ell} \in\{ \pm 1\}$ is the product of local signs around each interior vertex; around an interior vertex the edge labels either read 1,2,3 or 1,3,2 in clockwise order, and the local sign is + or accordingly,

- and $M(\ell)$ denotes a certain monomial in the $x_{i j}$ 's; an edge in $T$ adjacent to boundary vertex $i$ and with edge label $j$ contributes a factor of $x_{i j}$ to $M(\ell)$.

The polynomial $[T]$ is always $\mathrm{SL}_{3}$-invariant, and using this fact it follows by classical invariant theory that $[T] \in \mathbb{C}[\hat{\mathrm{Gr}}(3, n)]$ as claimed. We model the addition in $\mathbb{C}[\hat{\mathrm{Gr}}(3, n)]$ by adding tensor diagrams formally, and we model the multiplication by superimposing diagrams.

Example 1. We can represent the Pl ucker coordinates $\Delta_{i j k} \in \mathbb{C}[\hat{\operatorname{Gr}}(3, n)]$ by a tripod $T$ connecting the boundary vertices $i, j, k$. The expression (2) describing the invariant $[T]$ associated to this tripod agrees with the usual definition of the determinant as a sum over permutations.

A web is called non-elliptic if it has no 2-cyles at the boundary, and if every interior face is bounded by at least 6 sides. We call an element $x \in \mathbb{C}[\hat{\operatorname{Gr}}(3, n)]$ a non-elliptic web invariant if $x=[W]$ for $W$ a non-elliptic web.

Theorem (Kuperberg [12]). The non-elliptic web invariants form a basis for $\mathbb{C}[\hat{\mathrm{Gr}}(3, n)]$. 
Furthermore, there is an algorithm for rewriting an arbitrary linear combination of tensor diagram invariants in terms of the basis of non-elliptic web invariants. This is done by repeatedly applying certain skein relations between tensor diagram invariants. These skein relations are diagrammatic moves replacing a tensor diagram $T$ by another tensor diagram $T^{\prime}$ (or sometimes a sum of several tensor diagrams) that defines the same invariant. The relations are local, meaning they replace a small fragment of a tensor diagram with another fragment, without changing how $T$ looks outside of the fragment. We illustrate applying such a skein relation by example.

Example 2. Consider the Plücker coordinates $\Delta_{124}, \Delta_{135} \in \mathbb{C}[\hat{\mathrm{Gr}}(3,5)]$. We represent their product $\Delta_{124} \Delta_{135}$ as a tensor diagram by superimposing two tripods. By applying a Kuperberg skein relation of the form

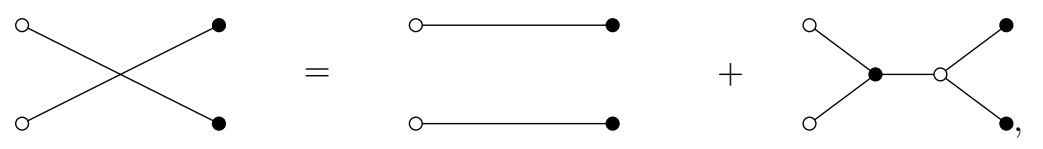

we can express $\Delta_{124} \Delta_{135}$ in the web basis as


The final combinatorial ingredient we will require is the arborization algorithm $W \mapsto \operatorname{Arb}(W)$ given by Fomin and Pylyavskyy [4]. It transforms a web $W$ by repeating the diagrammatic move in Figure 2 , as many times as this move is possible. Performing this move does not change the value of the invariant defined by $W$, i.e. the resulting tensor diagram $\operatorname{Arb}(W)$ satisfies $[\operatorname{Arb}(W)]=[W]$.

A non-elliptic web invariant $[W]$ is arborizable if $\operatorname{Arb}(W)$ has no internal cycles, and is indecomposable if $\operatorname{Arb}(W)$ is not a union of several tensor diagrams.
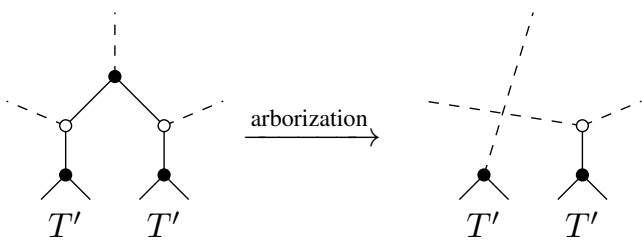

Fig. 2: An arborization step indicated schematically. One can apply this step whenever a given tensor diagram $T$ has two copies of the same binary tree $T^{\prime} \subset T$ connecting to the boundary, and these two copies of $T^{\prime}$ are joined by a path of length 4 . This is indicated schematically in the left portion of the figure. The dashed lines show how these two copies of $T^{\prime}$ are connected to the rest of $T$. The arborization step removes the path of length 4 and connects the two copies of $T^{\prime}$ to the rest of the diagram as indicated on the right hand side of the figure. 
Conjecture 1 (Fomin-Pylyavskyy [4, Sections 9 and 10]). The set of cluster (and frozen) variables in $\mathbb{C}[\hat{\mathrm{Gr}}(3, n)]$ coincides with the set of indecomposable non-elliptic web invariants that are arborizable. Two such cluster variables lie in the same cluster if and only if their product is again a non-elliptic web invariant.

Example 3. The web $W$ in Figure 1 is equal to its own arborization; there are no arborization steps to apply. However, this web is not arborizable since it has an interior cycle. On the other hand, every Plücker coordinate is an example of an indecomposable arborizable web.

The third web in (4) is not indecomposable. It arborizes as a product of two tripods, namely the ones for $\Delta_{134}$ and $\Delta_{125}$. Thus, (4) expresses the familiar Plücker relation

$$
\Delta_{124} \Delta_{135}=\Delta_{123} \Delta_{145}+\Delta_{134} \Delta_{125}
$$

in terms tensor diagram calculus.

As we have said, when $n \leq 8$, Conjecture 1 can be verified by hand since there are only finitely many cluster variables in these cases. We can now state our first two main results. We say two non-elliptic webs are compatible if their product is a non-elliptic invariant. This is the condition for two cluster variables to be in the same cluster according Conjectures 1.

Theorem 1. The set of cluster variables in $\mathbb{C}[\hat{\mathrm{Gr}}(3,9)]$ includes infinitely many indecomposable arborizable web invariants. The set of clusters includes infinitely many clusters whose cluster variables are pairwise compatible.

Computing cluster variables in $\mathbb{C}[\hat{\mathrm{Gr}}(3,9)]$ by performing mutations is not practical at the moment since there is no tensor diagram analogue for division. To compute the new cluster variable $x_{k}^{\prime}$ in a cluster variable exchange relation (1), it would seem one must first "guess" what $x_{k}^{\prime}$ must be, superimpose the tensor diagrams for $x_{k}$ and $x_{k}^{\prime}$, and then see if a sequence of skein relations produces the two monomials on the right hand side of (1). Theorem 1 and its proof indicate a way to construct complicated cluster variables without ever performing such mutation steps.

Theorem 2. The set of indecomposable web invariants in $\mathbb{C}[\hat{\mathrm{G}} \mathrm{r}(3,9)]$ includes infinitely many nonarborizable invariants.

Theorem 2 is relevant in light of the expected link between cluster algebras and canonical bases. A cluster monomial in a cluster algebra is a monomial in the elements of any extended cluster in the corresponding seed pattern. It is has long been suspected that cluster monomials should be a part of a naturally defined basis for the cluster algebra. Defining this canonical basis using the cluster structure is a guiding problem in the theory of cluster algebras. Recent breakthroughs by several groups [2, 10, 11] have reinforced the importance of this problem and suggested potential answers - for example it is now known that the set of cluster monomials is linearly independent. The nonarborizable webs in Theorem 2 are not cluster monomials. Consequently these webs should provide a distinguished role for understanding the relation between the various canonical bases and the cluster structure.

\section{A quasi-isomorphism with Fock-Goncharov space}

To prove Theorems 1 and Theorem 2, we will construct a group of symmetries of the cluster structure on $\mathbb{C}[\hat{\operatorname{Gr}}(3,9)]$. To find these symmetries, we will make use of a second family of cluster algebras that has received considerable interest in its own right. 
Fock and Goncharov have described a cluster algebra for each choice of semisimple Lie groups $G$ and marked bordered surfaces $S$. We will be interested in the case where $G=\mathrm{SL}_{3}$ and $S$ is a disk with $r$ marked points on its boundary. Let $V$ be a three-dimensional vector space.

Definition (Affine flags in $\left.\mathrm{SL}_{3}\right)$. An affine flag for $\mathrm{SL}_{3}$ is a pair $(v, v \wedge w) \in V \times \Lambda^{2}(V)$ for some $v, w \in V$ such that $v \wedge w \neq 0$. A configuration of $r$ affine flags is an $r$-tuple $\left(F_{1}, \ldots, F_{r}\right)$ of affine flags considered up to simultaneous action by $\mathrm{SL}_{3}$. The space of such configurations is denoted $\operatorname{Conf}_{r}\left(\mathrm{SL}_{3}\right)$.

The Fock-Goncharov cluster algebra occupying our interest is the ring $\mathbb{C}\left[\mathrm{Conf}{ }_{r}\left(\mathrm{SL}_{3}\right)\right]$ of polynomial functions on configurations of $r$ affine flags. by By appropriately modifying Definition 3 , the ring $\mathbb{C}\left[\operatorname{Conf}_{r}\left(\mathrm{SL}_{3}\right)\right]$ has its own version of web combinatorics obtained. Rather than placing $n$ black vertices on the boundary as we did for $\hat{\operatorname{Gr}}(3, n)$, the $r$ boundary vertices in a web for $\mathbb{C}\left[\operatorname{Conf}_{r}\left(\mathrm{SL}_{3}\right)\right]$ are thought of as being simultaneously black and white. One can define invariants $[W] \in \mathbb{C}\left[\mathrm{Conf}_{r}\left(\mathrm{SL}_{3}\right)\right]$, skein relations, and an arborization algorithm for webs in this context. Fomin and Pylyavskyy give an appropriate version of Conjecture 1 describing the cluster variables and clusters in $\mathbb{C}\left[\operatorname{Conf}_{r}\left(\mathrm{SL}_{3}\right)\right]$.

We now indicate the promised bijection between cluster variables in $\mathbb{C}[\hat{\mathrm{Gr}}(3,3 r)]$ and $\mathbb{C}\left[\mathrm{Conf}_{2 r}\left(\mathrm{SL}_{3}\right)\right]$. We will indicate the bijection by example in the case $r=2$ using Figure 3 . For a tensor diagram in $\mathbb{C}[\hat{\mathrm{Gr}}(3,6)]$, we obtain a tensor diagram for $\mathbb{C}\left[\mathrm{Conf}_{4}\left(\mathrm{SL}_{3}\right)\right]$ by "plugging" $T$ into the 6 black vertices produced in Figure 3 Similarly, we produce a tensor diagram for $\mathbb{C}[\hat{\mathrm{Gr}}(3,6)]$ from a web for $\mathbb{C}\left[\mathrm{Conf}_{4}\left(\mathrm{SL}_{3}\right)\right]$ by plugging into the 8 vertices ( 4 black and 4 white) in Figure 3 The $F_{11}$ and $F_{12}$ indicate the "black" and "white" arguments of the affine flag $F_{1}$ respectively. In both cases, if $T$ plugs in to a given boundary vertex more than once, the recipe requires plugging $T$ into several copies of the corresponding fragment in Figure 3
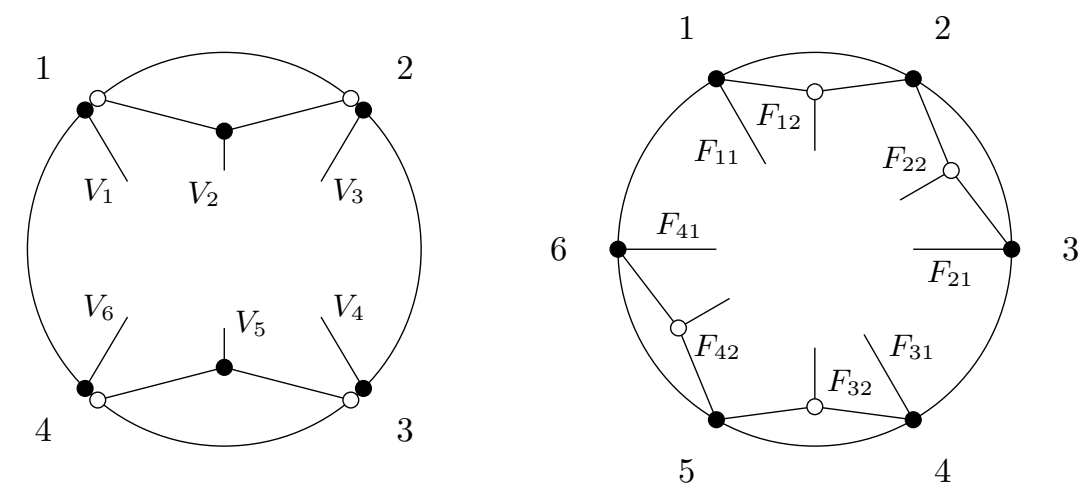

Fig. 3: The diagram on the left indicates schematically how to produce a tensor diagram for Conf $4\left(\mathrm{SL}_{3}\right)$ from a tensor diagram for $\operatorname{Gr}(3,6)$. This is done by plugging in the vectors $V_{1}, \ldots, V_{6}$ to the black strands in the figure. The right figure indicates how to produce a tensor diagram for $\mathrm{Gr}(3,6)$ from a tensor diagram for Conf $4\left(\mathrm{SL}_{3}\right)$. The arguments $F_{11}$ and $F_{12}$ indicate the black and white parts of the first affine flag and so on.

Example 4. Plugging in the Plücker coordinate $\Delta_{135}$ to the left diagram in Figure 3 produces a "quadrapod web" for Conf ${ }_{4}\left(\mathrm{SL}_{3}\right)$. It is obtained by joining the boundary vertices 1 and 2 for $\operatorname{Conf}_{4}\left(\mathrm{SL}_{3}\right)$ by a 
white fork, joining the boundary vertices 3 and 4 by a black fork, and then connecting these two forks to each other.

The rules in Figure 3 induce maps of algebras

$$
\begin{aligned}
& \Psi^{*}: \mathbb{C}\left[\operatorname{Conf}_{2 r}\left(\mathrm{SL}_{3}\right)\right] \rightarrow \mathbb{C}[\hat{\mathrm{Gr}}(3,3 r)] \\
& \Phi^{*}: \mathbb{C}[\hat{\mathrm{Gr}}(3,3 r)] \rightarrow \mathbb{C}\left[\operatorname{Conf}_{2 r}\left(\mathrm{SL}_{3}\right)\right]
\end{aligned}
$$

We have the following pair of parallel propositions, which we view as evidence for Conjecture 1 .

Proposition 3. If $x \in \mathbb{C}[\hat{\operatorname{Gr}}(3,3 r)]$ is a cluster variable then $\Phi^{*}(x)$ factors as $M x^{\prime}$ where $M$ is a monomial in the frozen variables for $\mathbb{C}\left[\mathrm{Conf}_{2 r}\left(S L_{3}\right)\right]$ and $x^{\prime}$ is a cluster variable. Ditto for $\Psi^{*}$ with the roles swapped. The map $x \mapsto x^{\prime}$ induces a bijection of the cluster variables and clusters in these two cluster algebras.

Proposition 4. Let $W$ be a non-elliptic web for $\operatorname{Gr}(3,3 r)$. Its image $\Phi^{*}([W]) \in \mathbb{C}\left[\operatorname{Conf}_{2 r}\left(\operatorname{SL}_{3}\right)\right]$ factors as $M\left[W^{\prime}\right]$ where $M$ is a monomial in the frozen variables for $\mathbb{C}\left[\right.$ Conf $\left.2_{2 r}\left(S L_{3}\right)\right]$ and $W^{\prime}$ is an indecomposable non-elliptic web. Furthermore, $W^{\prime}$ is arborizable if and only if $W$ is. The same statement holds for $\Psi^{*}$ with the roles of $\mathrm{Gr}$ and Conf swapped.

Proposition 3 is established algebraically. It follows from the statement that the algebra maps $\Phi^{*}$ and $\Psi^{*}$ describe a quasi-isomorphism between this pair of cluster algebras. The concept of a quasi-isomorphism (and related notions of quasi-homomorphisms and quasi-automorphisms) are studied systematically in [8]. Proposition 4 is proved combinatorially by analyzing the arborization steps that take place when evaluating the algebra homomorphisms $\Phi^{*}$ and $\Psi^{*}$. One also must check that the tensor diagram $W^{\prime}$ obtained by factoring out frozen variables is indeed a web invariant (i.e., that it can be given by a planar diagram). In fact, the map $W \mapsto W^{\prime}$ can be described explicitly in terms of web combinatorics.

Now we leverage the maps $\Phi^{*}$ and $\Psi^{*}$ to create otherwise hidden symmetries of the Grassmannian.

Definition (Cyclic shifts and duality). Let $P$ denote the map on $\mathbb{C}\left[\operatorname{Conf}_{r}\left(\mathrm{SL}_{3}\right)\right]$ induced by cyclic rotation of affine flags

$$
\left(F_{1}, \ldots, F_{r}\right) \stackrel{P}{\mapsto}\left(F_{2}, \ldots, F_{r}, F_{1}\right) .
$$

Thus $P$ acts on webs for $\operatorname{Conf}_{r}\left(\mathrm{SL}_{3}\right)$ by rotating the $r$ boundary vertices clockwise. Let $D$ denote the map on webs for $\mathbb{C}\left[\operatorname{Conf}_{r}\left(\mathrm{SL}_{3}\right)\right]$ that globally swaps the black and white colors. Finally, let $\rho$ denote the cyclic shift on $\mathbb{C}[\operatorname{Gr}(3, n)]$ induced by rotating the $n$ boundary vertices.

The cluster structure on $\mathbb{C}\left[\operatorname{Conf}_{r}\left(\mathrm{SL}_{3}\right)\right]$ is equivariant with respect to the symmetries $P$ and $D$. However, intertwining with the maps $\Phi^{*}$ and $\Psi^{*}, P$ and $D$ produces new symmetries of the cluster structure on $\mathbb{C}[\hat{\operatorname{Gr}}(3,3 r)]$. By Proposition 3 , these maps induce permutations of the cluster variables for $\operatorname{Gr}(3,3 r)$. We denote these permutations by $\dot{P}$ and $\dot{D}$, and we let $\dot{\rho}$ denote the permutation corresponding to $\rho$. By Proposition 4, we can think of these elements acting on all indecomposable webs (not only on cluster variables). 
Theorem 5. Suppose $r \geq 3$ and $k=3$. The elements $\dot{P}, \dot{D}$, and $\dot{\rho}$ satisfy the relations

$$
\begin{aligned}
\dot{D}^{2}=\dot{\rho}^{r k} & =1 \\
\dot{\rho}^{k} & =\dot{P}^{2} \\
\dot{P} \dot{D} & =\dot{D} \dot{P} \\
\dot{\rho} \dot{P} \dot{D} & =\dot{P} \dot{D} \dot{\rho},
\end{aligned}
$$

and these relations give a presentation for the group these elements generate.

The group presented by the relations in in Theorem 5 has exponential growth. The proof of Theorems 1. 2. and 5 goes by analyzing the action of the elements $\dot{P}, \dot{D}$, and $\dot{\rho}$ on particular webs, using the explicit description of the maps $\Phi^{*}$ and $\Psi^{*}$. A variant of the Ping Pong Lemma coupled with this explicit description can be used to show that the relations in Theorem 9 give a presentation.

To prove Theorem 2 we consider the orbit of the nonarborizable web in Figure 1 (see also Example 3) with respect to the group in Theorem 5. One can show that orbit is infinite using a Ping Pong Lemma argument. Acting on a Plücker coordinate (or on a cluster consisting entirely of Plücker coordinates) and checking the orbit is infinite, one obtains Theorem 1 .

\section{Webs in higher rank}

There is a generalization of Definition 3 that comes from replacing $\mathrm{SL}_{3}$ by $\mathrm{SL}_{k}$ with $k$ arbitrary . The underlying combinatorial object is as follows:

Definition. A web for $S L_{k}$ is a finite bipartite graph drawn in the disk with $n$ boundary black vertices, with edges labeled by positive integers in $[1, k-1]$, subject to two additional requirements. First, every interior vertex is either bivalent or trivalent, and the sum of the edge labels around each interior vertex is $k$. Second, every edge that connects to the boundary is labeled with a 1 .

In a similar fashion to 2 , one can try to define an invariant $[W] \in \mathbb{C}[\hat{\mathrm{G}} \mathrm{r}(k, n)]$ corresponding to a web $W$. Unfortunately, there is no natural choice of the local signs around an interior vertex, and the invariant $[W]$ is only well-defined up to a choice of \pm sign. There are ways of fixing this ambiguity using directed edges and tags [1], but we will suppress this technicality to streamline our discussion.

When $G=\mathrm{SL}_{k}$, the Fock-Goncharov cluster algebra is a ring $\mathbb{C}\left[\mathrm{Conf}_{r}\left(\mathrm{SL}_{k}\right)\right]$ of polynomial functions on configurations of $r$ affine flags in $S L_{k}$. To define webs for this cluster algebra, one simply drops the requirement that all boundary edges have weight 1 in Definition 5

Cautis, Kamnizer, and Morrison have described a complete set of local diagrammmatic relations amongst $\mathrm{SL}_{k}$ web invariants [1]. However the combinatorics is more complicated when $k>3$. One reflection of this is that there is not yet a simple notion for what it should mean for an $\mathrm{SL}_{k}$-web to be non-elliptic, i.e. there is no natural basis for $\mathbb{C}[\hat{\mathrm{Gr}}(k, n)]$ consisting of web invariants when $k \geq 4$. There is no arborization algorithm (or even a proposal for one), and no version of Conjecture 1 . On the other hand, there is hope that one can use $\mathrm{SL}_{k}$ webs to study the cluster combinatorics for the cluster algebras $\mathbb{C}[\hat{\mathrm{Gr}}(k, n)]$ and $\mathbb{C}\left[\operatorname{Conf}_{r}\left(\mathrm{SL}_{k}\right)\right]$ associated with $G=\mathrm{SL}_{k}$. We are able to make some progress towards this hope.

Theorem 6. The maps $\Phi^{*}$ and $\Psi^{*}$ bear appropriate generalizations to algebra maps $\Psi^{*}: \mathbb{C}\left[\operatorname{Conf}_{2 r}\left(S L_{k}\right)\right] \rightarrow$ $\mathbb{C}[\hat{\mathrm{Gr}}(k, r k)]$ and $\Phi^{*}: \mathbb{C}[\hat{\mathrm{Gr}}(k, r k)] \rightarrow \mathbb{C}\left[\right.$ Conf $\left._{2 r}\left(S L_{k}\right)\right]$. These maps provide a quasi-isomorphism of the corresponding cluster algebras. If $[W] \in \mathbb{C}\left[\right.$ Conf $\left._{2 r}\left(S L_{k}\right)\right]$ is a web invariant, then so is $\Psi^{*}([W]) \in$ $\mathbb{C}[\hat{\mathrm{Gr}}(k, r k)]$, and ditto for $\Phi^{*}$. 
For arbitary $k$, one can make sense of the cyclic shift and duality maps $\rho, P$ and $D$. One obtains certain symmetries of the cluster combinatorics for $\mathbb{C}[\hat{\mathrm{Gr}}(k, r k)]$. The relations in Theorem 5 still hold and we conjecture that they still give a presentation. Acting by these symmetries on Plücker coordinates, we get a large class of cluster variables for $\mathbb{C}[\hat{\mathrm{Gr}}(k, r k)]$ all of which are web invariants by Theorem 6 .

Finally, we would like to highlight a connection between our and previous work. Marsh and Scott have given a version of a twist map for the cluster structure on $\mathbb{C}[\hat{\mathrm{Gr}}(k, n)]$, cf. [13] and [14]. This twist map can be recovered from our group of symmetries (in the case $k$ divides $n$ ):

Theorem 7. Let $F$ be the algebra endomorphism of $\mathbb{C}[\hat{\mathrm{G}} \mathrm{r}(k, r k)]$ defined by intertwining $P^{-1} \circ D$ with the quasi-isomorphisms $\Phi^{*}$ and $\Psi^{*}$ from Equation 6 Then $F$ agrees with the twist map of Marsh and Scott.

\section{Further remarks}

There are several directions which remain to be explored. In particular, we make the following conjecture.

Conjecture 2. The set of nonarborizable indecomposable webs for $\mathbb{C}[\hat{\mathrm{Gr}}(3,9)]$ is precisely the orbit of the web in Figure 1 under the action of the group in Theorem 5.

Fock and Goncharov have defined the cluster modular group; it is an abstractly defined group of symmetries of a cluster algebra that is difficult to compute in practice. The group in Theorem 5 is a subgroup of the cluster modular group for $\mathbb{C}[\hat{\mathrm{Gr}}(3,9)]$. We speculate that these two groups might coincide.

\section{Acknowledgements}

I thank Dylan Thurston, Greg Mueller, and especially Ian Le for helpful conversations. I thank my advisor Sergey Fomin for proposing this line of inquiry and for many conversations and suggestions.

\section{References}

[1] S. Cautis, J. Kamnitzer,S. Morrison, Webs and Quantum Skew Howe Duality, Mathematische Annalen, 1-40, 2012.

[2] G. Cerulli Irelli, B. Keller, D. Labardini-Fragoso, P.-G. Plamondon, Linear independence of cluster monomials for skew-symmetric cluster algebras, Compos. Math. 149 (2013), no. 10, 1753-1764.

[3] V. V. Fock and A. B. Goncharov, Moduli spaces of local systems and higher Teichmüller Theory, Publ. Math. Inst. Hautes. Etudes. Sci. no. 103 (2006) 1-211.

[4] S. Fomin and P. Pylyavskyy, Tensor diagrams and cluster algebras, arXiv:1210.1888.

[5] S. Fomin and P. Pylyavskyy, Webs on surfaces, rings of invariants, and cluster algebras, Proc. Natl. Acad. Sci. USA no. textbf111 (2014), no. 27, 9680-9687.

[6] S. Fomin and D. Thurston, Cluster algebras and triangulated surfaces. Part II: Lambda lengths, arXiv: 1210.5569 .

[7] S. Fomin and A. Zelevinsky, Cluster algebras I: Foundations, J. Amer. Math. Soc. 15 (2002), no. 2, 497-529 
[8] C. Fraser, Quasi-homomorphisms of cluster algebras, arXiv:1509.05385.

[9] M. Gehktman, M. Shapiro, and A. Vainshtein, Cluster algebras and Poisson geometry, Mosc. Math. J. 3 (2003), no. 3, 899-934.

[10] M. Gross, P. Hacking, S. Keel, M. Kontsevich, Canonical bases for cluster algebras, arXiv:1411.1394.

[11] S. J. Kang, M. Kashiwara, M. Kim, S.-j. Oh, Monoidal categorification of cluster algebras II, arXiv:1502.06714.

[12] G. Kuperberg, Spiders for rank 2 Lie algebras, Comm. Math. Phys. 180 (1996), 109-151.

[13] R. J. Marsh, J. Scott, Twists of Plücker coordinates as dimer partition functions, arXiv:1309.6630.

[14] G. Muller and D. Speyer. The twist for positroid varieties, 2015, (forthcoming).

[15] J. Scott, Grassmannians and cluster algebras, Proc. London Math. Soc. (3) 92 (2006), no. 2, 345380. 
